\title{
Identifying bleeding: Go with the flow
}

\author{
M. U. Callaghan (10)
}

Division of Pediatric Hematology/Oncology, Carmen and Ann Adams Department of Pediatrics, Children's Hospital of Michigan, Wayne State University, Detroit, MI, USA

\section{Correspondence}

Michael U. Callaghan, Division of Pediatric Hematology/Oncology, Carmen and Ann Adams Department of Pediatrics, Children's Hospital of Michigan, Wayne State University, Detroit, MI, USA.

Email: mcallagh@med.wayne.edu

In this issue of Haemophilia, the manuscript from Yaoi et al. describes the use of a new microfluidic platform (t-TAS) to examine bleeding associated with severe anaemia. ${ }^{1}$ The new tool has advantages over most traditional compartmental and global coagulation assays in that it evaluates platelet thrombus formation under conditions of flow and shear stress. In doing so, they demonstrate the importance of red blood cells (RBCs) in haemostasis. Specifically, they posit that RBCs act to physically marginate platelets to the vessel wall and enable platelet activation and thrombus formation under high shear conditions. We have often noted prolonged PFA-100 (a test that also involves flow and very high shear) times in anaemic patients but long considered this an artefact or limitation of the test. In this study, the authors used t-TAS to measure thrombus formation under high and very high shear conditions and demonstrate that this tool correlated with the clinical findings in a young woman with type I mild von Willebrand disease with menorrhagia. The patient continued to bleed and have an abnormal t-TAS in spite of full correction of von Willebrand factor (VWF) levels but stopped bleeding after packed RBC transfusion. They went on to demonstrate using ex vivo-manipulated samples that t-TAS was sensitive to both VWF levels and haematocrit.

As we enter the postgenomic era, laboratory tools to monitor bleeding phenotype have only become more important. Genetics and molecular biology have given us great insight into bleeding phenomena but approaching unanswered questions will require detailed mechanistic understanding of coagulation and better phenotyping. Better phenotyping will rely on new tools such as t-TAS that allow us to examine the role of heretofore unevaluated but important factors in haemostasis including dynamic measures under physiologic conditions and the role of endothelium.

I recently came across a book, entitled The Sports Gene, ${ }^{2}$ that reports on the role of genetics in athletic performance. In the first chapter, the author relays a story about major league baseball's best hitters and Jennifer Finch, the star pitcher for the USA Olympic Softball team. Albert Pujols, Barry Bonds and other all stars were unable to hit her pitches. It turns out that the assumption that outstanding hitters rely on coordination and trigger fast reflexes was wrong. Outstanding hitters recognize patterns of arm movement and baseball rotation very quickly upon release of a pitch and superior recognition guides their hitting. Further studies identified that superior visual acuity, perception and ability to track moving objects are more important to hitting than reaction time or bat speed. An examination of the Los Angeles Dodgers team revealed that more than half of the Dodgers position players had visual acuity of $20 / 10$ or better (which fewer than $1 \%$ of the general population have). If we were to look for genetic or mechanistic biology underlying good hitting based on reaction times or bat speed, we would be unlikely to find it. Only by identifying that superior visual acuity defines the phenotype of good hitting could we get to the biology. Similarly, if we define laboratory phenotypes based on components of haemostasis without flow condition, we would miss the important role of RBCs.

The complex interplay between genes, non-coding DNA, epigenetics and environment constitute so many variables that systematically evaluating them from an unbiased perspective exceeds capacity of subjects in the world and they must be evaluated in relation to phenotypes which are often inaccurate, incomplete or mischaracterized. In Dickens' A Christmas Carol, Ebeneezer Scrooge is visited by the ghosts of Christmas-past, present and future. Modern science is now visited by the legends of haematology-past, present and future. The legends of haematology past used thorough clinical evaluation to identify specific phenotypes and separate them into disorders that allow us to test interventions and improve care. The legends of haematology present have used powerful genetic and molecular biology tools to identify the underlying defects behind these phenotypes and target these defects to improve care. After the completely penetrant monogenic and deterministic environmental causes of disease have been determined through state of the art genetic and molecular biology evaluation, there will remain many important unanswered questions. The legends of haematology future will need to incorporate mechanistic gene and protein level understanding of biology into complex physiologically 
driven models, combining this with new tools and understanding of complex phenotypes to improve our understanding of haematologic diseases and improve care for our patients moving forward. Yaoi et al. have re-examined the laboratory phenotype in bleeding patients using an important new tool that incorporates flow and shear enabling us to identify factors and further characterize bleeding phenotypes previously unrecognized in the clinical laboratory.

\section{DISCLOSURES}

The authors stated that they had no interests which might be perceived as posing a conflict or bias.

\section{REFERENCES}

1. Yaoi H, Shida Y, Ogiwara K, Hosokawa K, Shima M, Nogami K. Role of red blood cells in the anemia-associated bleeding under high shear conditions. Haemophilia. 2017, https://doi.org/10.1111/hae.13252.

2. Epstein DJ. The Sports Gene: What Makes the Perfect Athlete. New York, USA: Penguin Books; 2013.

How to cite this article: Callaghan MU. Identifying bleeding: Go with the flow. Haemophilia. 2017;23:652-653. https://doi.org/10.1111/hae.13304 